\title{
The COVID-19 immune landscape is dynamically and reversibly correlated with disease severity
}

\author{
Hamid Bolouri, ${ }^{1,2}$ Cate Speake, ${ }^{3}$ David Skibinski, ${ }^{3}$ S. Alice Long, ${ }^{4}$ Anne M. Hocking, ${ }^{4}$ Daniel J. Campbell, ${ }^{5,6}$ Jessica A. Hamerman, ${ }^{5,6}$ \\ Uma Malhotra, ${ }^{7,8}$ Jane H. Buckner, ${ }^{4,6}$ and the Benaroya Research Institute COVID-19 Research Team ${ }^{9}$ \\ 'Center for Systems Immunology, Benaroya Research Institute (BRI) at Virginia Mason, Seattle, Washington, USA. ${ }^{2}$ Division of Human Biology, Fred Hutchinson Cancer Research Center, Seattle, Washington, \\ USA. ${ }^{3}$ Center for Interventional Immunology, ${ }^{4}$ Center for Translational Immunology, and ${ }^{5}$ Center for Fundamental Immunology, Benaroya Research Institute (BRI) at Virginia Mason, Seattle, Washington, \\ USA. ${ }^{6}$ Department of Immunology, University of Washington School of Medicine, Seattle, Washington, USA. Department of Infectious Disease, Virginia Mason Medical Center, Seattle, Washington, USA. \\ ${ }^{8}$ Department of Medicine, Section of Infectious Diseases, University of Washington, Seattle, Washington, USA. ${ }^{9}$ The BRI COVID-19 Research Team is detailed in the supplemental acknowledgments.
}

BACKCROUND. Despite a rapidly growing body of literature on coronavirus disease 2019 (COVID-19), our understanding of the immune correlates of disease severity, course, and outcome remains poor.

\begin{abstract}
METHODS. Using mass cytometry, we assessed the immune landscape in longitudinal whole-blood specimens from 59 patients presenting with acute COVID-19 and classified based on maximal disease severity. Hospitalized patients negative for SARS-CoV-2 were used as controls.
\end{abstract}

RESULTS. We found that the immune landscape in COVID-19 formed 3 dominant clusters, which correlated with disease severity. Longitudinal analysis identified a pattern of productive innate and adaptive immune responses in individuals who had a moderate disease course, whereas those with severe disease had features suggestive of a protracted and dysregulated immune response. Further, we identified coordinate immune alterations accompanying clinical improvement and decline that were also seen in patients who received IL-6 pathway blockade.

CONCLUSION. The hospitalized COVID-19 negative cohort allowed us to identify immune alterations that were shared between severe COVID-19 and other critically ill patients. Collectively, our findings indicate that selection of immune interventions should be based in part on disease presentation and early disease trajectory due to the profound differences in the immune response in those with mild to moderate disease and those with the most severe disease.

FUNDING. Benaroya Family Foundation, the Leonard and Norma Klorfine Foundation, Clenn and Mary Lynn Mounger, and the National Institutes of Health.

\section{Introduction}

The coronavirus disease 2019 (COVID-19) pandemic has brought a worldwide focus not only on the severe acute respiratory syndrome coronavirus 2 (SARS-CoV-2), but also on how immunity to the virus both promotes viral clearance and contributes to morbidity and mortality in infected individuals. There is a wide range of disease severity in SARS-CoV-2-infected individuals, ranging from asymptomatic infection to severe COVID-19 requiring mechanical ventilation, and in some cases, to death. Some factors have been identified that are associated with increased disease severity and poor outcome during COVID-19,

Conflict of interest: JHB is president of the Benaroya Research Institute at Virginia Mason, scientific cofounder and scientific advisory board member of CentiBio, consultant for Bristol-Myers Squibb, and has past and current research projects sponsored by Amgen, Bristol-Myers Squibb, Janssen, Novo Nordisk, and Pfizer. JHB also has a patent, no: US8053235B2, "Methods of generating antigen-specific CD4+CD25 regulatory T cells, compositions and methods of use." Copyright: (c) 2021, American Society for Clinical Investigation.

Submitted: August 25, 2020; Accepted: November 25, 2020; Published: February 1, 2021 Reference information: J Clin Invest. 2021;131(3):e143648.

https://doi.org/10.1172/JCl143648. including age, race, obesity, hypertension, and type 2 diabetes (1-11). However, we still do not understand the biologic factors that contribute to disease severity and outcome. It is becoming clear that not only does the severity of disease vary among SARS-CoV-2-infected individuals, but the immune response can also vary widely, leading to differing immune landscapes between patients. Therefore, it is important to understand how the immune landscape contributes to COVID-19 severity and outcome. Another important gap in our knowledge is how the immune landscape in COVID-19 resembles or is distinct from that seen in critically ill patients hospitalized for other reasons, since the immune landscape may change in the context of critical illness regardless of its etiology. In particular, it is important to determine if the early immune landscape can be used to inform which COVID-19 patients will have a severe disease course and would benefit from early interventions.

Although we can learn about immunity to SARS-CoV-2 by assessing a snapshot of the immune response at one point in time, the immune response to infection is dynamic and is best studied over time. Early immune responses to viruses are dominated by the innate immune system, including neutrophils, 


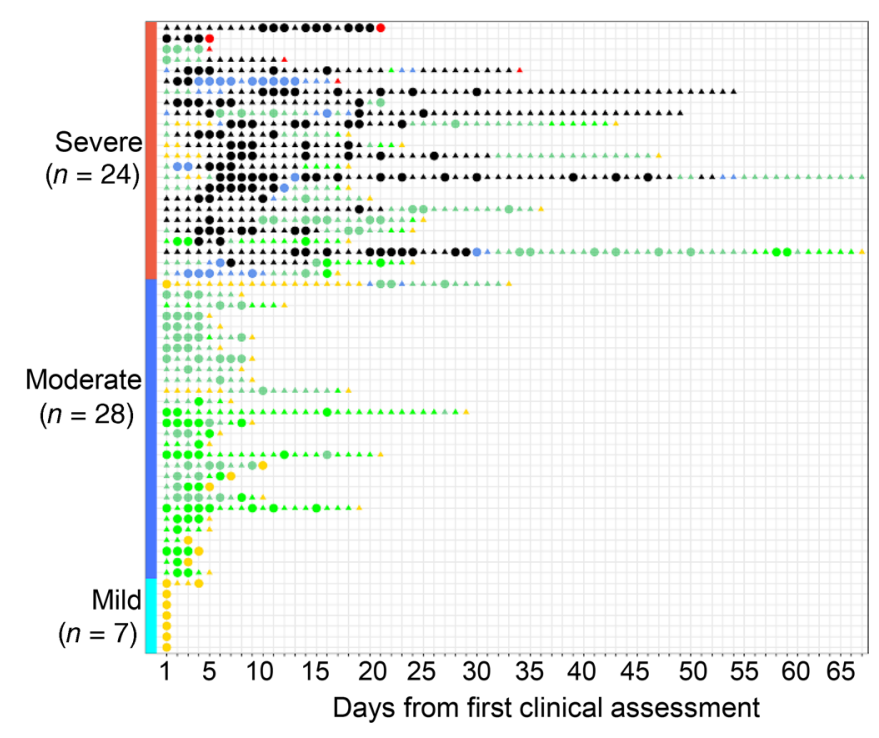

Disease ordinal score

2, not hospitalized but unable to resume normal activities

3 , hospitalized, not requiring supplemental oxygen

4, hospitalized, requiring supplemental oxygen

5 , hospitalized, requiring nasal high flow oxygen therapy, non-invasive mechanical ventilation or both

6, hospitalized, requiring invasive mechanical ventilation 7 , death

monocytes, plasmacytoid dendritic cells (pDCs), and natural killer (NK) cells, whereas adaptive immune responses of $\mathrm{T}$ and B cells critical for viral clearance develop over days to weeks. Understanding how these populations change over time and relate to disease trajectory can give insight into the signature of a productive anti-SARS-CoV-2 immune response associated with clinical improvement, and whether immune dysregulation contributes to severe COVID-19. Additionally, early in the pandemic hospitalized patients were treated with a variety of experimental therapeutics, including the antiviral agent remdesivir, cytokine modulating therapies, and plasma from convalescent patients, all with varying efficacy in clinical studies and trials. However, how and if these treatments affect the immune landscape before and after therapeutic exposure has not been described. To address these outstanding and important questions regarding the immune response during COVID-19, we used mass cytometry integrated with detailed clinical data to examine how the immune landscape changes over time in severe and moderate disease through natural progression and recovery, and also in the context of immune intervention.

\section{Results}

Patient demographics and clinical characteristics. We collected peripheral blood from 59 patients with COVID-19 (52 hospitalized patients and 7 ambulatory outpatients) at the Virginia Mason Medical Center, Seattle, Washington, USA, during the months of April and May 2020. Notably, we performed deep longitudinal sampling over the course of disease with an average of 4 time points per subject (range: 1-18; Figure 1), allowing for detailed immune trajectories of recovery. Patients were
Figure 1. Clinical course and mechanistic data for subjects with COVID-19. Each subject is represented in one row. Subjects are first grouped by severity: severe (red), moderate (blue), and mild (cyan) disease. Subjects are next ranked by highest-ever ordinal score (most severe at top) and finally by minimum ordinal score (representing the largest change over time). The $x$ axis shows days from first clinical assessment, typically the date of hospital admission. Colored points represent the ordinal score captured daily. No subjects had a score of 1 (recovered) at any point. Dates with CyTOF data available are denoted by circles; dates without CyTOF data are denoted by triangles.

classified based on maximum disease severity using a 7-point ordinal scale (OS) representing the following outcomes: 1 , not hospitalized with resumption of normal activities; 2, not hospitalized, but unable to resume normal activities; 3 , hospitalized, not requiring supplemental oxygen; 4, hospitalized, requiring supplemental oxygen; 5 , hospitalized, requiring nasal high-flow oxygen therapy, noninvasive mechanical ventilation, or both; 6 , hospitalized, invasive mechanical ventilation; and 7, death (12). Of the hospitalized patients, 24 were classified as having severe disease on the basis of requiring management in a critical care unit (CCU); all required mechanical ventilation (maximal OS $\geq$ 6 ), except one who was on high-flow oxygen (maximal OS =5). The remaining 28 hospitalized patients were not in the CCU and were classified as having moderate COVID-19, with all requiring supplemental oxygen at some point in their hospital course (maximal OS $=3-5$ ). The 7 ambulatory patients had mild disease $(\mathrm{OS}=2)$ and did not require hospitalization. For a control group, we also collected blood from 17 hospitalized patients who tested negative for SARS-CoV-2; 4 of these patients were admitted to the $\mathrm{CCU}$ and the remainder to the floor. These patients were ageand sex-matched to the hospitalized COVID-19 groups and were admitted for a variety of conditions including respiratory $(n=4)$, cardiac $(n=4)$, gastrointestinal $(n=3)$, neurologic $(n=3)$, and miscellaneous conditions $(n=3)$.

The demographic and clinical characteristics of all the patient groups are summarized in Table 1. There was no significant difference in age or sex composition between severe, moderate, and mild COVID-19 groups. Regarding racial distribution, there was an overrepresentation in the severe COVID-19 group of African American (16.7\%) and Hispanic (37.5\%) individuals based on the Washington state population, which is $78.5 \%$ White, $4.4 \%$ African American, and 13\% Hispanic (13). Duration of symptoms at time of presentation was longer in the severe disease group (median 9 days, range 3-22) compared with both the moderate (median 4 days, range $0-27$ ) and mild (median 5 days, range 2-14) groups ( $P$ $=0.01$ ). Duration of hospitalization was also significantly longer in the severe disease group (median 19 days, range 4-65) compared with the moderate disease group (median 6 days, range 2-28) ( $P$ $<0.01$ ), although discharge was delayed for some patients due to restrictions placed on transfers to skilled nursing facility pending viral clearance from nasopharyngeal swabs.

Chronic medical conditions such as diabetes, hypertension, and cancer were common in the hospitalized COVID-19 cohorts. Diabetes was present in $45.8 \%$ of the severe group, $28.6 \%$ of the moderate group, and $28.6 \%$ of the mild group. Hypertension was present in $50 \%$ of the severe group and $67.9 \%$ of the moderate 
Table 1. Cohort demographics and clinical characteristics

\begin{tabular}{|c|c|c|c|c|}
\hline & $\begin{array}{l}\text { Severe } \\
\text { COVID-19 } \\
(n=24)\end{array}$ & $\begin{array}{l}\text { Moderate } \\
\text { COVID-19 } \\
(n=28)\end{array}$ & $\begin{array}{c}\text { Mild } \\
\text { COVID-19 } \\
(n=7)\end{array}$ & $\begin{array}{l}\text { Hospitalized } \\
\text { COVID-19 negative } \\
(n=17)\end{array}$ \\
\hline & Median (range) & Median (range) & Median (range) & Median (range) \\
\hline Age, years & $61(31-89)$ & $67(34-96)$ & $54(27-76)$ & $67(30-97)$ \\
\hline Number of days hospitalized & $19(4-65)$ & $6(2-28)$ & NA & $5(1-34)$ \\
\hline Days from symptom onset to admission & $9(3-22)$ & $4(0-27)$ & $5(2-14)$ & NA \\
\hline \multirow[t]{2}{*}{ Disease score at admission } & $6(3-6)$ & $4(2-4)$ & & \\
\hline & $2(2)$ & $4(3-4)$ & & \\
\hline \multirow[t]{2}{*}{ BMI } & $30.1(18.1-54.5)$ & $29.0(17.0-54.1)$ & $25.5(22.5-37.2)$ & $25.7(21.1-69)$ \\
\hline & Number (\%) & Number (\%) & Number (\%) & Number (\%) \\
\hline \multicolumn{5}{|l|}{ Outcome } \\
\hline Discharged & $18(75)$ & $28(100)$ & NA & $16(94.1)$ \\
\hline Deceased & $6(25)$ & $0(0)$ & NA & $1(5.9)$ \\
\hline Female & $12(50)$ & $14(50)$ & $3(42.9)$ & $10(58.8)$ \\
\hline \multicolumn{5}{|l|}{ Race/ethnicity } \\
\hline Asian & $1(4.2)$ & $3(10.7)$ & $3(42.9)$ & $1(5.9)$ \\
\hline African American & $4(16.7)$ & $5(17.9)$ & $1(14.3)$ & $0(0)$ \\
\hline Native Hawaiian/Pacific Islander & $0(0)$ & $1(3.6)$ & $0(0)$ & $0(0)$ \\
\hline Native American & $2(8.3)$ & $0(0)$ & $0(0)$ & $1(5.9)$ \\
\hline White & $6(25)$ & $17(60.7)$ & $2(28.6)$ & $14(73.7)$ \\
\hline Unknown/Other & $11(45.8)$ & $2(7.1)$ & $1(14.3)$ & $1(5.9)$ \\
\hline Hispanic/Latino & $9(37.5)$ & $2(7.1)$ & $1(14.3)$ & $0(0)$ \\
\hline \multicolumn{5}{|l|}{ Preexisting comorbidities } \\
\hline Cancer & $1(4.2)$ & $6(21.4)$ & $0(0)$ & $2(8.7)$ \\
\hline Diabetes & $11(45.8)$ & $8(28.6)$ & $2(28.6)$ & $3(17.6)$ \\
\hline Hypertension & $12(50)$ & $19(67.9)$ & $0(0)$ & $6(35.3)$ \\
\hline \multicolumn{5}{|l|}{ Exposure to experimental medicine } \\
\hline Hydroxychloroquine & $7(29.2)$ & $2(7.1)$ & $0(0)$ & NA \\
\hline Remdesivir & $17(70.8)$ & $11(39.3)$ & $0(0)$ & NA \\
\hline Tocilizumab & $8(33.3)$ & $0(0)$ & $0(0)$ & NA \\
\hline Convalescent plasma & $15(62.5)$ & $4(14.3)$ & $0(0)$ & NA \\
\hline
\end{tabular}

Elevated white blood cell counts in COVID-19 correlate with increased neutrophils and decreased plasmacytoid dendritic cells and basophils. We assessed the immune landscape by combining clinical data with mass cytometry (CyTOF) performed on whole blood samples recovered from the clinical laboratory. The CyTOF panel was designed to assess the composition of the innate and lymphocyte compartments and determine the maturation, lineage, and activation status of these cell populations (Supplemental Table 1 and Supplemental Figures 2-4). To better understand the impact of disease, we performed a correlation analysis on the first sample collected for each patient in the COVID-19 cohort ( $n$ = 59; Figure 2 and Supplemental Figure 5). The heatmap in Figure 2A shows all significant correlations between clinical data (disease severity ordinal score, age, BMI, and $\mathrm{CBC}$ ) and CyTOF immune cell percentages of the total CD45 $5^{+}$(pan-leukocyte marker) cell compartment, whereas the correlation network in Figure 2B focuses only on correlations among major leukocyte populations identified by CyTOF. We found correlations consistent with the current literature. For example, WBC counts and neutrophil counts were significantly correlated (Figure 2A), not surprisingly given that neutrophils comprise a large proportion of WBC, and both are elevated in severe COVID-19 $(14,15)$. Neutrophils in both the CBC and CyTOF data sets also inversely correlated with group but absent in the mild group. Cancer was present in $4.2 \%$ of the severe COVID-19 group, $21.4 \%$ of the moderate group, and absent in the mild group. Obesity was also more prevalent in the hospitalized COVID-19 cohort with a median BMI greater than 29 in both severe and moderate disease groups compared with a median BMI of approximately 25 in the mild COVID-19 $(P=0.08)$ and the hospitalized SARS-CoV-2-negative groups.

Because this cohort was from the early stage of the pandemic in the USA, hospitalized patients received a variety of experimental treatments, including hydroxychloroquine, remdesivir, tocilizumab, and convalescent plasma (Supplemental Figure 1; supplemental material available online with this article; https:// doi.org/10.1172/JCI143648DS1). Notably, many patients received more than one type of experimental treatment. In the severe COVID-19 group, 7 patients (29.2\%) received hydroxychloroquine, $17(70.8 \%)$ received remdesivir, 8 (33.3\%) received tocilizumab, and $15(62.5 \%)$ received convalescent plasma. Among the moderately ill, 2 (7.1\%) received hydroxychloroquine, 11 (39.3\%) received remdesivir, and 4 (14.3\%) received convalescent plasma. The mild disease group did not receive any of these COVID-19 therapies. proportions of lymphocytes and T cells (Figure 2, A and B) supporting previous reports that the neutrophil-to-lymphocyte ratio is increased in severe COVID-19 (15-18). In addition, both pDCs and basophils negatively correlated with neutrophils, positively correlated with $\mathrm{T}$ cells, and positively correlated with each other (Figure 3, A-E). Together, these findings for pDCs and basophils are consistent with recent studies reporting depletion of these cell types in acute COVID-19 (19, 20). Although our CyTOF panel had limited ability to distinguish $\mathrm{T}$ cell lineage, $\mathrm{T}$ follicular helper (Tfh) cells were assessed. Notably, unlike other T cell populations, the percentage of Tfh cells in the memory $\mathrm{CD} 4^{+}$compartment showed a positive correlation with neutrophils, although this did not reach statistical significance (Figure 3F). Taken together, these observations indicate that coordinate and counter-acting changes in neutrophils, lymphocytes, pDCs, and basophils contribute to the immune signature of COVID-19.

The immune landscape differentiates individuals based on disease severity. In order to understand whether the immune signature in COVID-19 differed by disease severity, we determined the correlation between cell frequency and ordinal score at the time of sam- 


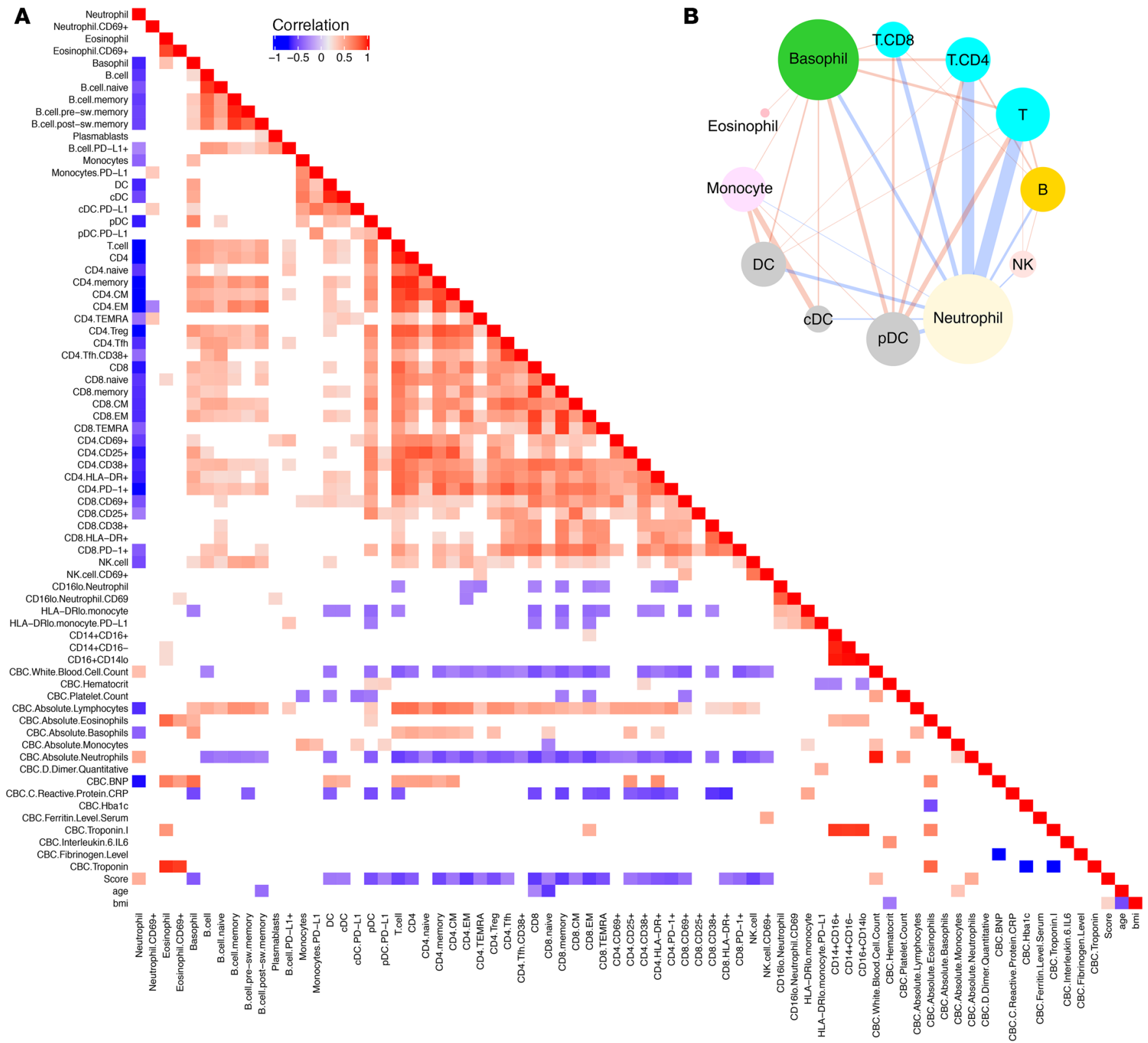

Figure 2. Overview of correlations among cell frequencies and COVID-19 patient characteristics. (A) Heatmap visualization of pairwise Pearson correlations with $P<0.05$ among ordinal score, age, BMI, CyTOF population frequencies, and CBC parameters. Key indicates $r$ value scale for positive (red) and negative (blue) correlations. (B) Network map visualization of correlations between CyTOF major immune cell subsets in our mild, moderate, and severe COVID-19 cohort. Shown are positive (red lines) and negative (blue lines) Pearson correlations with absolute $(r)>0.35$ and $P<0.05$. Line thickness corresponds to the strength of association (thicker is stronger). Correlations within major cell populations (same-color nodes) are not shown.

pling. Increasing neutrophil frequency was positively correlated with increasing disease severity (Pearson correlation $\sim 0.46$, FDR-adjusted $P<0.01$ ), whereas T cells, NK cells, pDCs, and basophils were lower in severe disease (all FDR-adjusted $P$ values $<0.005$, Figure 4 ). To determine if the immune landscape early in disease distinguishes severe from mild disease, we next performed a cross-sectional analysis of our population categorized based on an individual's highest disease score during the course of their illness using data from the first sample collected for each patient (Figure 5, Supplemental Figure 5). The CBC data showed the greatest difference with disease severity in WBC counts with an increase in the absolute neutrophils and monocyte counts and low absolute lymphocyte counts (Figure $5 \mathrm{~A})$. However, these $\mathrm{CBC}$ results frequently fell within the normal range and notably, the hospitalized COVID-19-negative population showed very similar changes to those seen with severe COVID-19, suggesting that these findings are not unique to COVID-19 but are instead reflective of critical illness.

In contrast, the cross-sectional analysis of the CyTOF data set identified 2 different patterns of immune alterations in the COVID-19 cohort: those that were also present in the hospitalized COVID-19-negative cohort and those that were unique to severe COVID-19. Immune cell populations that were similar between 


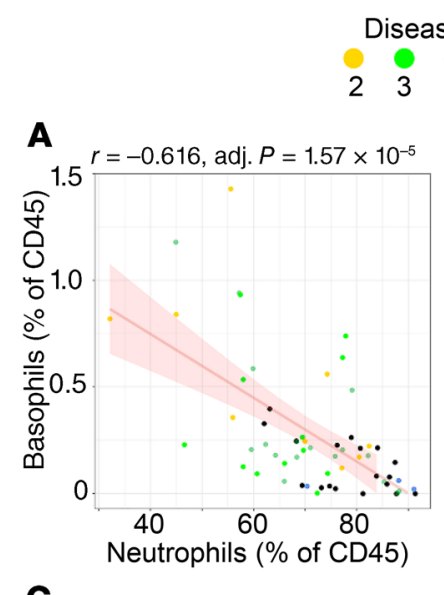

C
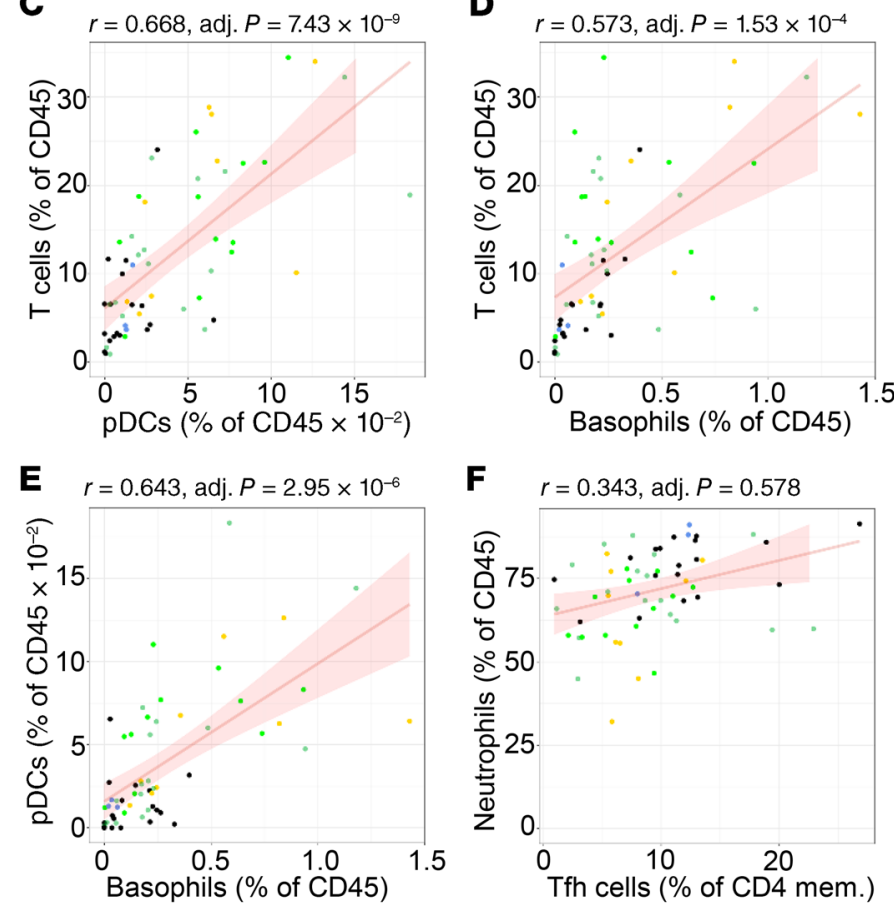

Figure 3. Correlations among immune cell populations in patients with COVID-19 demonstrate a relationship between disease severity and an increase in neutrophils and a depletion of pDCs and basophils. (A-F) Plots display FDR-adjusted Pearson correlations and linear regression lines with $95 \%$ confidence interval shading. Data points are colored according to the ordinal score observed for each patient at admission.

erally differentiated individuals based on their disease severity, with more moderate disease courses and good outcome associated with clusters A and B, while those with the most severe disease and death were associated with cluster $\mathrm{C}$. These findings indicate that there is not one single immune signature in COVID-19, but that the immune response differs in individuals based on the ultimate disease severity.

Immune trajectories discriminate moderate and severe COVID-19. To better understand the kinetics and coordinated changes in immune signatures, we tracked immune cell types in the blood over time based on date of admittance to the hospital. We focused on exploring differences in longitudinal analysis of moderate and severe patients based on distinct clustering between these groups as shown in Figure 6. The time course was limited to 15 days after admittance for sufficient and comparable sampling in both the moderate and severe cohorts (Supplemental Figure 6).

Key to understanding features that distinguish moderate from severe COVID-19 is an appreciation of the evolution of the immune response over time, as shown in a uniform manifold approximation and projection (UMAP) visualization of immune changes with disease severity within cell types of an individual patient (Figure 7). Using gated data from Figures 2-7, we focused on specific cell types and markers of innate and adaptive immunity. Figure 8 shows Loess-smoothed trajectories whereas Supplemental Figures 7-9 show individual and averaged plots. We found that patients with moderate COVID-19 had a dynamic immune response that resolved over time, typical of a productive antiviral response, whereas patients with severe COVID-19 had an aberrant immune response, diverging early from that seen in moderate COVID-19 subjects and continuing to diverge beyond the first 15 days of hospitalization. Specifically in the moderate COVID-19 cohort, there was an early reduction in circulating neutrophils with a concomitant increase in circulating monocytes, total DCs, and basophils, with maximal change at $4-5$ days after hospitalization (Figure 8A and Supplemental Figures 7-9). In addition, NK cells increased followed these early myeloid cell changes, peaking at 5-6 days after hospitalization (Figure 8A and Supplemental Figures 8 and 9). In contrast with the severe COVID-19 cohort, these innate cell populations were less dynamic with little variation during the first 15 days of hospitalization (Figure 8A and Supplemental Figures 8 and 9). However, it should be noted that this was not the case for all innate cells examined. For example, HLA-DR ${ }^{10}$ monocytes, which we and others found to be increased in severe COVID-19 (Figure 5B and ref. 21) and which are known to be increased in severe inflammatory syndromes such as sepsis (22-24), were more dynamic in the severe COVID-19 cohort than in the moderate COVID-19 cohort. HLA-DR ${ }^{10}$ monocytes in subjects with severe COVID-19 increased with time, peaking at 5-6 days of hospital- 
A Neutrophils

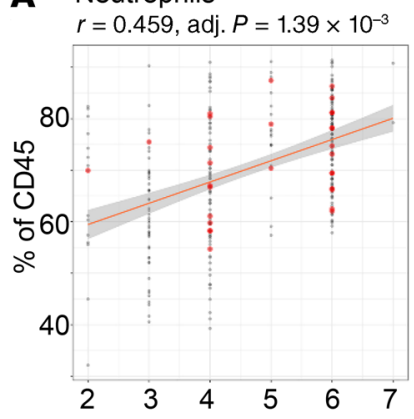

C NK cells
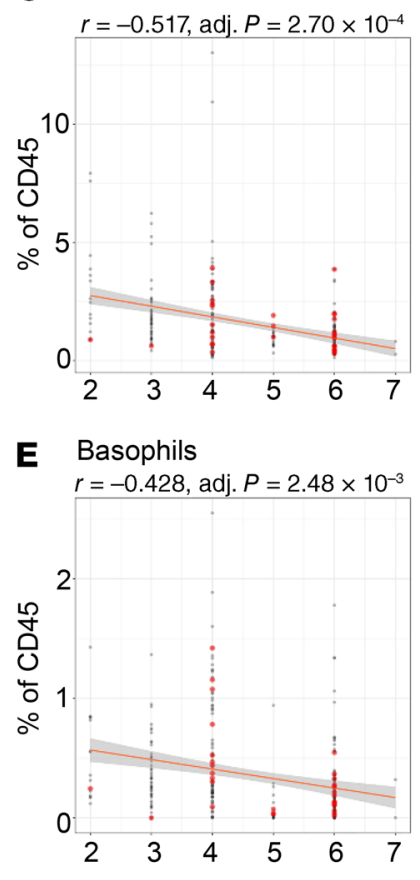

B T cells

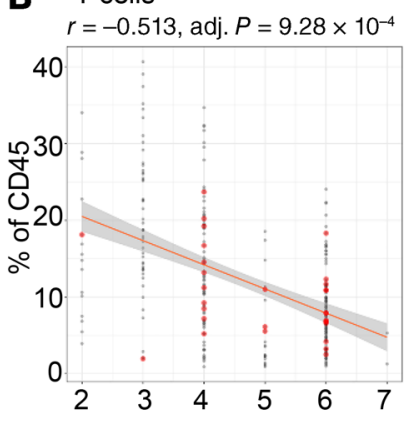

D $\mathrm{pDCs}$

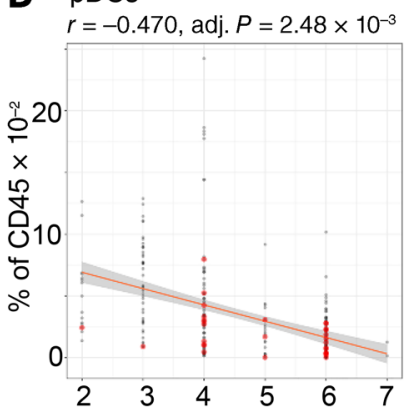

gestive of sustained interaction with memory $\mathrm{CD} 4^{+} \mathrm{T}$ cells and antibody production. In contrast, patients with severe COVID-19 consistently had lower levels of both $\mathrm{T}$ cells and memory B cells over the course of hospitalization, suggesting a diminished or delayed adaptive immune response to the virus. The Tfh response in the severe COVID-19 cohort was greater than that of the moderate COVID-19 cohort at all time points, perhaps indicating unresolved T cell help or Tfh cells sustained by high IL- 6 in critically ill patients. In addition, in the severe COVID-19 cohort, Treg cells as a percentage of total $\mathrm{CD} 4^{+} \mathrm{T}$ cells were increased over time as compared with the moderate COVID-19 cohort (Figure 8B), likely in response to ongoing inflammation due to viral persistence. Consistent with this idea, the percentage of $\mathrm{CD} 8^{+} \mathrm{T}$ cells expressing HLA-DR, a marker of activation, also increased over time in the severe COVID-19 cohort (Figure 8B), as did CD8 $8^{+} \mathrm{T}$ cells expressing CD38 and PD-1 (Supplemental Figure 7B), whereas total memory $\mathrm{CD} 8^{+} \mathrm{T}$ cell increases were similar between moderate and severe patients (Figure 8B). Overall, our longitudinal analysis revealed that the immune trajectory differs between moderate and severe patients during the first 2 weeks after initial hospitalization. Patients with moderate disease showed signatures of a productive antiviral response that resolved within the 2 weeks of the study time, whereas patients with severe disease showed signs of an aberrant response after hospital admittance that persisted for at least the first 2 weeks in hospital.

Immune signatures of clinical improvement in patients with COVID-19. To identify key immune cell populations that are associated with either clinical improvement or decline, we focused our analysis on samples taken from individuals before and after a change in ordinal score, reflective of disease severity. We assessed changes in the absolute abundance of immune cell populations by $\mathrm{CBC}$ or in the frequency of immune cell subsets in our CyTOF analyses across these key clinical times. We identified subjects that had samples drawn across a score improvement of 2 or more, or a score decline of 1 or more (Figure 9A). This analysis identified several populations whose abundance or frequency was significantly altered upon changes in ordinal score (Figure 9B). Consistent with the lymphopenia observed in severe COVID-19, we found that absolute lymphocytes decreased with clinical decline whereas an increase in the absolute number of lymphocytes was associated with clinical improvement. The increase in lymphocytes was mediated by a general increase in the frequency of naive and memory $\mathrm{CD}^{+}$and $\mathrm{CD} 8^{+} \mathrm{T}$ cells as well as NK cells, but not $\mathrm{B}$ cells. The frequency of pDCs also increased in subjects around the time of clinical improvement, whereas the frequency of neutrophils decreased in improving patients. Longitudinal analysis of individual subjects further demonstrated that changes in the frequency of neutrophils, T cells, NK cells, and pDCs could be observed during recovery from severe COVID-19 (Figure 9C). This analysis demonstrates that the immune landscape is dynamic in COVID-19, and that resolution of key features of severe disease is coincident with improvement in clinical status.

Early immune signatures of tocilizumab, but not convalescent plasma, treatment in patients with severe COVID-19. To determine if there were immune signatures of tocilizumab or convalescent plasma treatment, we identified 7 patients treated with tocilizum$\mathrm{ab}$ and 7 patients treated with convalescent plasma in our cohort 
A

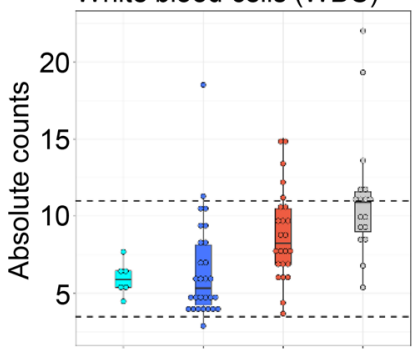

B
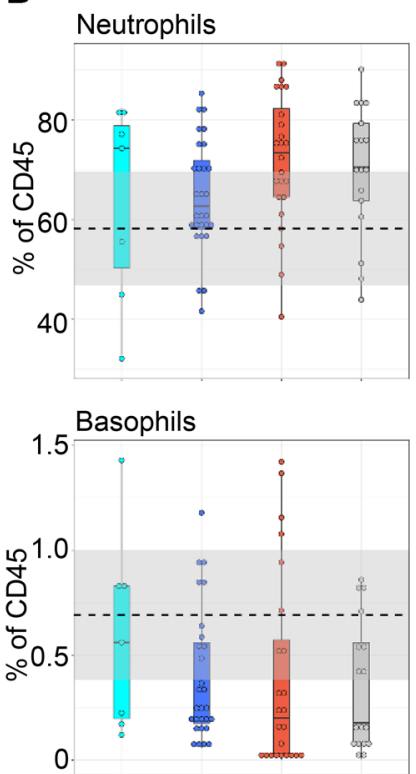

C

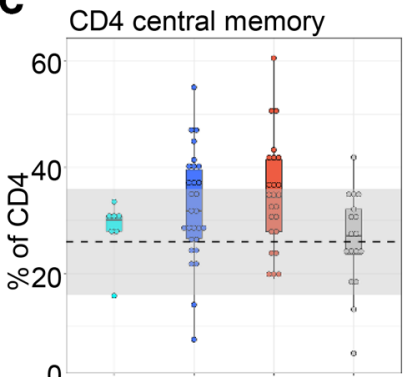

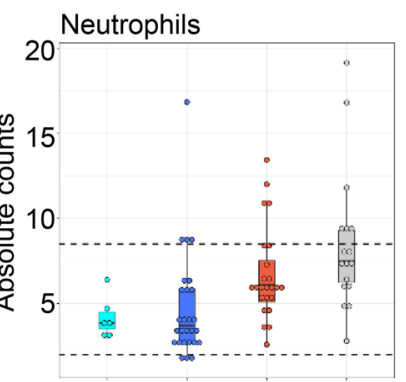
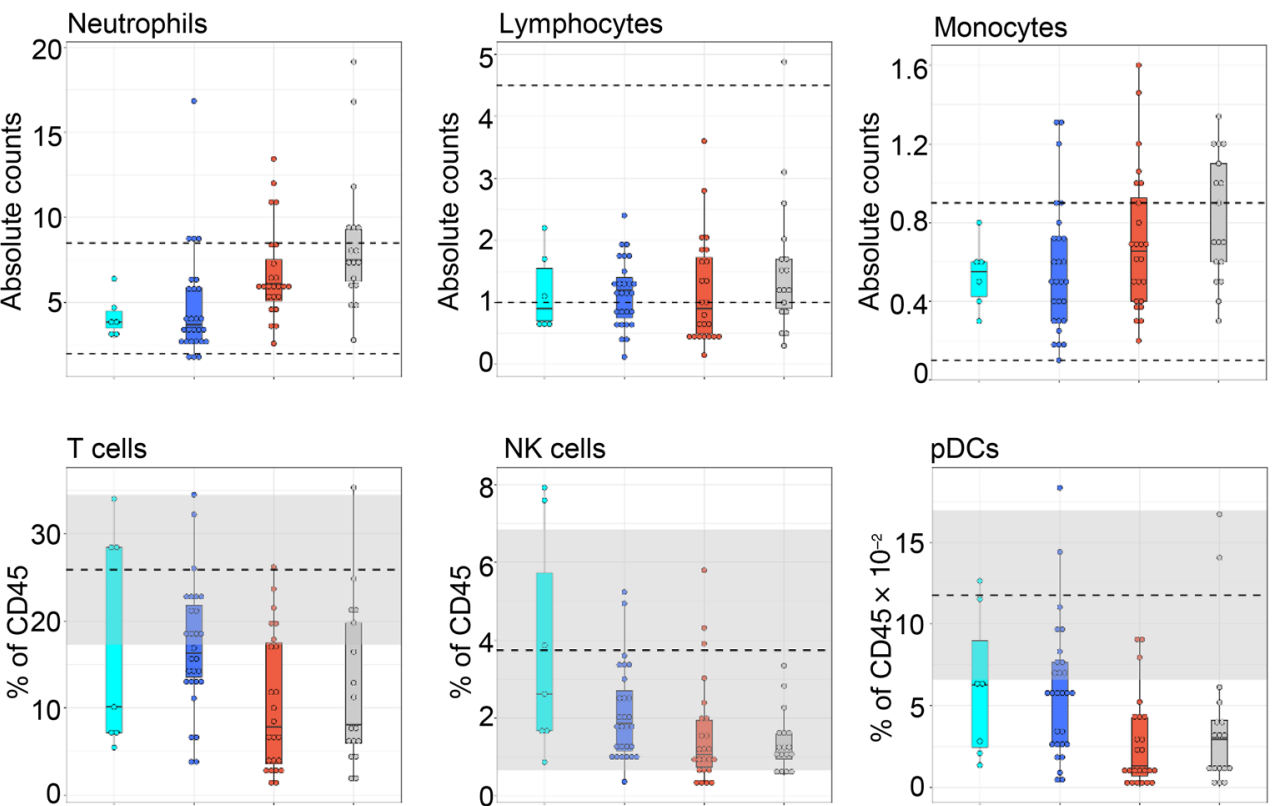

\section{HLA-DR Monocytes}
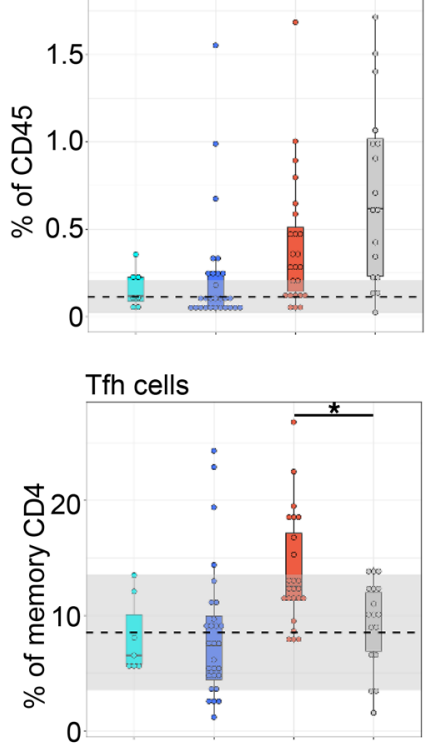
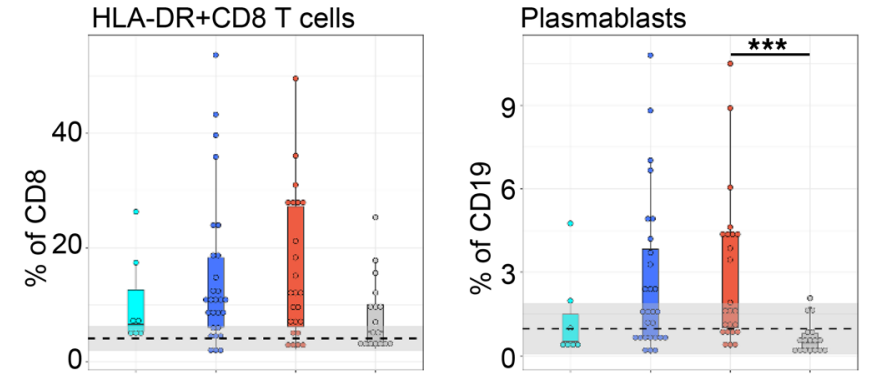

Figure 5. Immune cell frequencies vary by COVID-19 disease severity. (A) Clinically measured CBC absolute count values from day of admission. Dashed black lines mark the clinical laboratories normal ranges. Subjects grouped based on disease severity, mild (cyan), moderate (blue), and severe (red), and SARS-CoV-2-negative hospitalized controls (gray). (B and C) The relative proportions of immune cell sub-types vary by disease severity. CyTOF cell frequencies based on disease severity expressed as either percentage of all leukocytes (B) or percentage of parent population (C). Gray bands mark the mean (dashed black line) $\pm 1 \mathrm{SD}$ in 20 healthy control subjects. ${ }^{*} P<0.05,{ }^{* * *} P<0.001$.

who had CyTOF samples both before and after treatment (Supplemental Tables 2 and 3). Notably, these patients all had severe disease and there were stringent criteria for the use of tocilizum$\mathrm{ab}$, including rapidly escalating oxygen needs combined with an IL-6 level greater than 20 times the upper limit of normal (ULN); and C-reactive protein (CRP) greater than $125 \mathrm{mg} / \mathrm{L}$ (ULN = 7.9). Marked elevations in ferritin, LDH, and D-dimer were also weighted in the decision-making process. All patients were also treated with remdesivir, with the exception of one patient in the convalescent plasma group. Additionally, 6 of 7 patients in the tocilizumab group analyzed were treated with convalescent plas- ma prior to tocilizumab treatment (1-6 days before tocilizumab). None of the 7 patients in the convalescent plasma group were treated with tocilizumab during the time points analyzed. Also important, patient care was similar between the 2 groups as the use of tocilizumab in our hospital does not result in alterations to patient care.

We first assessed serum CRP levels in these 2 groups as a measure of the effectiveness of tocilizumab treatment, which should reduce this marker of systemic inflammation. Indeed, treatment with tocilizumab swiftly reduced serum CRP in all patients (Figure 6A). Serum ferritin was also reduced mainly 


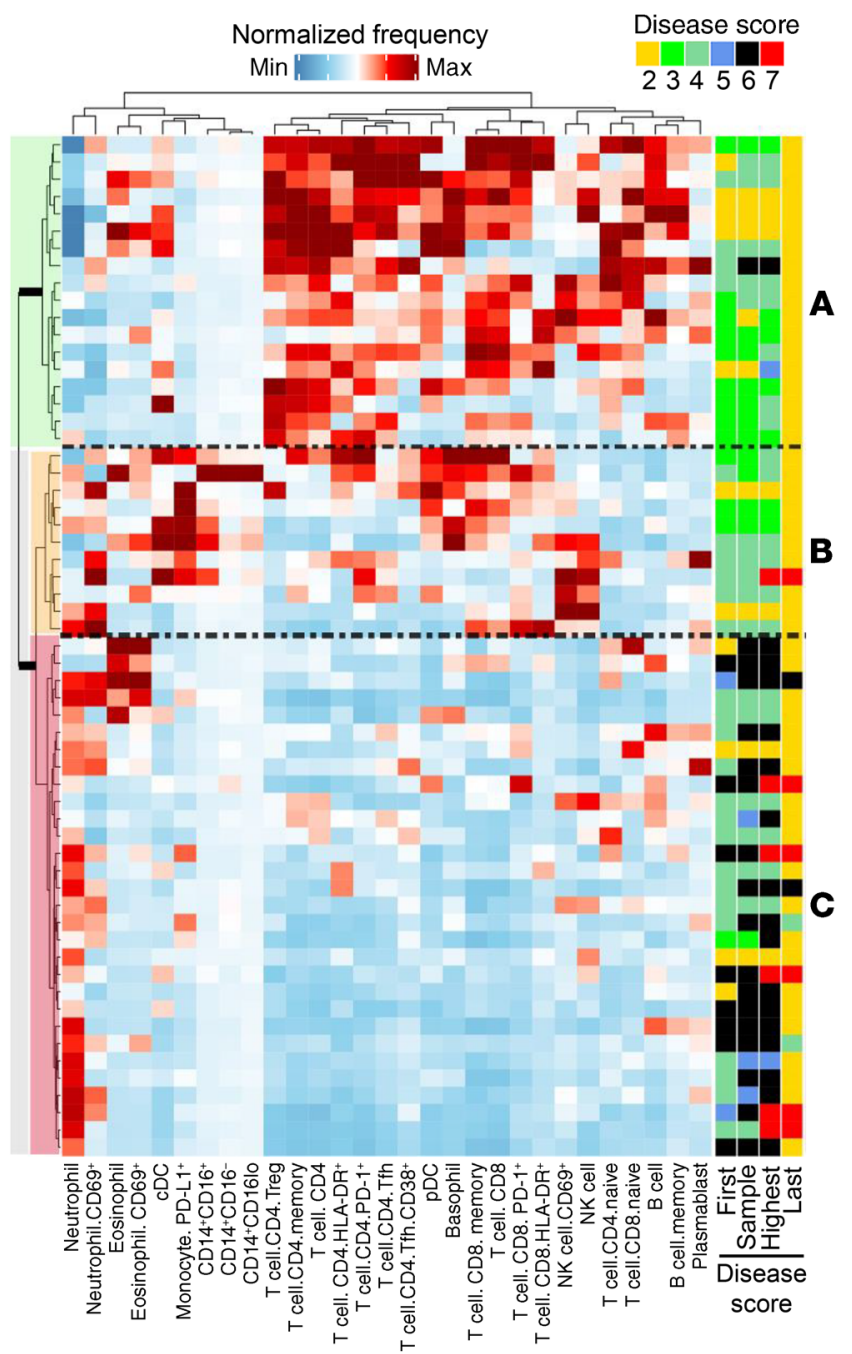

Figure 6. Admission day sample CyTOF cell frequencies. The admission day sample CYTOF cell frequencies fall into 3 distinct clusters. The heatmap shows row-normalized $z$ scores thresholded at \pm 2 (see color key). Disease severity scores are shown on the right side of the heatmap for the day of admission, day of sampling, maximum score, and score at discharge (disease score key shown at top of heatmap). Clusters are marked (A-C) at right, and indicated by green, orange, and red highlighting on the dendrogram at left.

in those patients with very high concentrations pretreatment (Supplemental Figure 10A). In contrast, convalescent plasma treatment had no consistent effect on CRP levels (Figure 10A). Therefore, tocilizumab treatment showed an acute clinical signature of reduced inflammation in patients with severe COVID-19, whereas convalescent plasma did not consistently affect these measures.

We then compared acute changes in immune populations in the blood before and after tocilizumab or convalescent plasma treatment by assessing the closest CyTOF sample before day of treatment (range: day -4 to day 0) with the first CyTOF sample available after treatment (range: day 2 to day 9 after treatment). The specific time points used for each individual are shown in Supplemental Table 3. Dividing the posttreatment time point cell frequency by the pretreatment time point for each patient allowed us to assess the fold change in response to treatment for each patient. In the tocilizumab group there were several populations of immune cells that differed significantly before and after treatment (Figure 10B). In contrast, there were no significant changes after convalescent plasma treatment in the immune cell populations analyzed by CyTOF (Figure $10 \mathrm{C}$ and Supplemental Figure $10, B$ and $C$ ). The significant changes in response to tocilizumab treatment included a reduction in the percentage of neutrophils and an increase in the percentage of total $\mathrm{T}$ cells, eosinophils, basophils, and DCs among CD45 cells (Figure 10, B, D, and E). There were also increases in several $\mathrm{CD}^{+}$and $\mathrm{CD} 8^{+} \mathrm{T}$ cell subpopulations, and no changes in any $\mathrm{B}$ cell populations after tocilizumab (Figure 10B). Moreover, our findings for T cells, B cells, neutrophils, and basophils were consistent with our signature of clinical improvement (Figure 9). However, there was not complete overlap between the tocilizumab signature and the clinical improvement signature, as NK cells and pDCs were not significantly changed by tocilizumab (Figure 10B, data not shown) but were increased in improving patients (Figure 10B). In the tocilizumab group, we also identified increased populations associated with $\mathrm{T}$ cell activation, including $\mathrm{HLA}-\mathrm{DR}^{+}$and $\mathrm{CD} 38^{+} \mathrm{CD} 4^{+}$and $\mathrm{CD}^{+} \mathrm{T}$ cells (Figure 10, B, D, and E). In summary, we observed a clear acute signature of tocilizumab treatment that shares some but not all features of the immunologic changes seen with clinical improvement, whereas there is no acute change in the immune landscape with convalescent plasma treatment, in patients with severe COVID-19.

\section{Discussion}

A growing body of literature indicates that the immune landscape is profoundly altered by COVID-19 and differs between individuals dependent on disease severity $(18,20,25,26)$. Whether the immune landscape is a reflection of disease severity, a source of severe disease, or a combination of the 2 is still not fully understood. Here, we utilized recovered samples from the clinical laboratory to rapidly assess peripheral blood cell populations by CyTOF and these data were analyzed in conjunction with clinical laboratories and disease severity scores. Importantly, we were able to collect samples longitudinally among the hospitalized individuals, allowing us to examine the evolution of immune responses through natural progression and recovery, and in the context of immune intervention. Novel aspects of our study included: (a) deep longitudinal sampling allowing for detailed immune trajectories of recovery, (b) a control cohort of moderate and severely ill hospitalized COVID-19 negative patients, and (c) analysis of immune signatures associated with tocilizumab and convalescent plasma treatments.

Notably, we found that at the time of initial sampling the immune landscape in COVID-19 forms 3 dominant clusters that relate to disease severity. When we examined individual cell populations based on disease severity, we found, as others have, that the neutrophil-to-lymphocyte ratio is increased in individuals with severe COVID-19 (15-18). Furthermore, this inverse relationship with neutrophils applies to basophils, DC, NK cells, and monocytes, and only modestly with B cells, and is most pronounced among $\mathrm{T}$ lymphocytes with the exception of Th cells, which are positively correlated to neutrophil numbers, a finding 

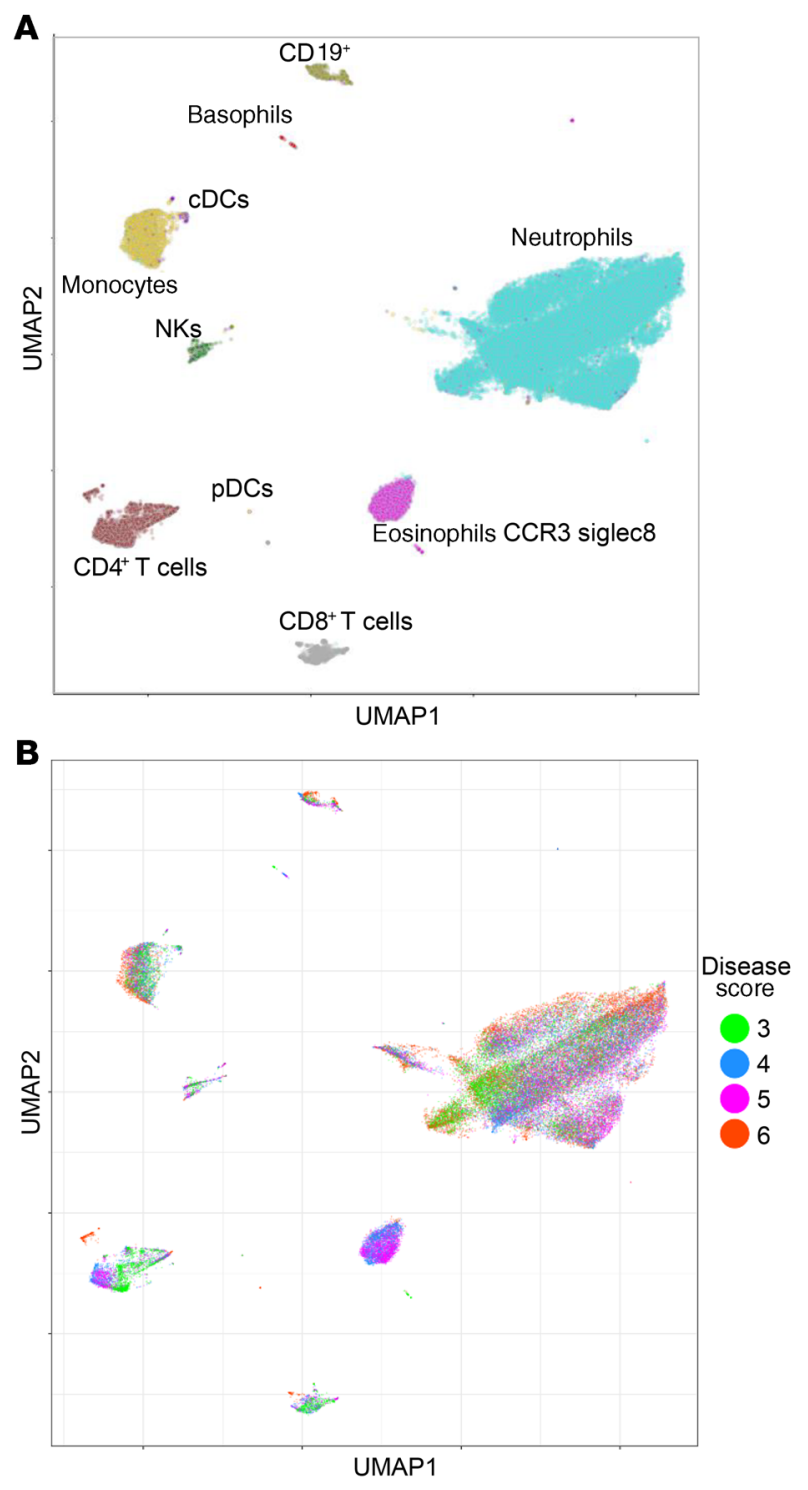

Figure 7. The COVID-19 immune landscape changes over recovery time. UMAP projections of batch-corrected CyTOF probe intensities for 4 samples from a single patient with COVID-19 recovering from a disease severity ordinal score of 6 to a score of 3 over a period of 6 weeks (see Methods for details).

also consistent with the current literature (18). Interestingly, many changes seen in severe COVID-19 compared with mild and moderate disease were also seen in our hospitalized COVID-19negative control cohort. Including this unique control group allowed us to identify differences between critically ill patients in general and those infected with SARS-CoV-2. Features shared between the patients hospitalized with severe COVID-19 and the COVID-19-negative cohort included increased neutrophils, and decreased T cells, NK cells, pDCs and basophils, and likely reflect active inflammation during critical illness. In contrast, increased Tfh cells, plasmablasts, and evidence of T cell activation were unique to the patients with severe COVID-19 and may reflect the antiviral response in these individuals or unique aspects of the pathology of SARS-CoV-2 infection.
Our longitudinal assessment further allowed us to identify patterns that distinguished severe and moderate disease. Individuals with a moderate disease course showed a pattern consistent with productive innate and adaptive immune response characterized by early and transient increases in monocytes and NK cells with later sustained increases in memory $\mathrm{T}$ and $\mathrm{B}$ cells. Those with severe disease have features suggestive of a dysregulated immune response characterized by delayed and prolonged increases in $\mathrm{Tfh}$ cells, HLA-DR ${ }^{\text {lo }}$ monocytes, and activated $\mathrm{CD} 8^{+} \mathrm{T}$ cells. Although the time from first symptom and first sample was delayed in severe patients as compared with moderate patients, this was not reflected in a simple shift of the immune trajectory. Instead, changes in multiple cell types of moderate subjects were transitory while evidence of persistent activation in different immune cells were progressive and unresolved in severe patients. This suggests that the degree of inflammation or persistence of virus markedly changed the immune landscape over time in severe as compared with moderate disease. Importantly, the persistent features of severe disease are reversed with improvements in clinical score and can be modulated in part with immune interventions, such as IL-6 pathway blockade.

Our findings for the tocilizumab study were intriguing, especially in the light of the recent disappointing results from the first randomized double blind phase 3 trial (27). Our data suggest that changes in the immune landscape after tocilizumab treatment, with the exception of NK cell and pDC recovery, are consistent with the immune signature of clinical improvement that we identified. Of note, only 4 of 7 of these tocilizumab-treated patients improved clinically in the acute time frame of our analysis (within 9 days of treatment) with the remaining 3 patients only showing clinical improvement at later times. This suggests that although tocilizumab treatment induces an acute signature of clinical improvement in both serum CRP and specific immune cell populations, this signature is disconnected from immediate clinical change, indicating that the immune changes with tocilizumab may be inadequate to support full recovery. Caveats for our tocilizumab analysis include the small cohort size, and that all but one of these patients were treated with convalescent plasma prior to tocilizumab treatment. Therefore, it is possible that convalescent plasma acts synergistically with tocilizumab to cause the immune signature we identified. Interestingly and in contrast to tocilizumab, we saw no clear immune signature of convalescent plasma within 7 days, suggesting either our cohort was too small to see changes, the immune populations change after the times we analyzed, or convalescent plasma does not act at the level of blood leukocyte populations. It is clear that further investigation is needed to determine whether tocilizumab has a therapeutic role in COVID-19, and in what patient population it would be useful, and this may be determined in part by the character and trajectory of the immune landscape of the patient.

The demographics of our patients with COVID-19 were consistent with published case reports. African Americans and Hispanics were overrepresented in the severe COVID-19 group relative to the population of Washington state, which is consistent with reports from other states in the USA $(10,11)$. We also found that type 2 diabetes was more common in those with severe disease compared with those with moderate or mild disease. Notably, all groups have 

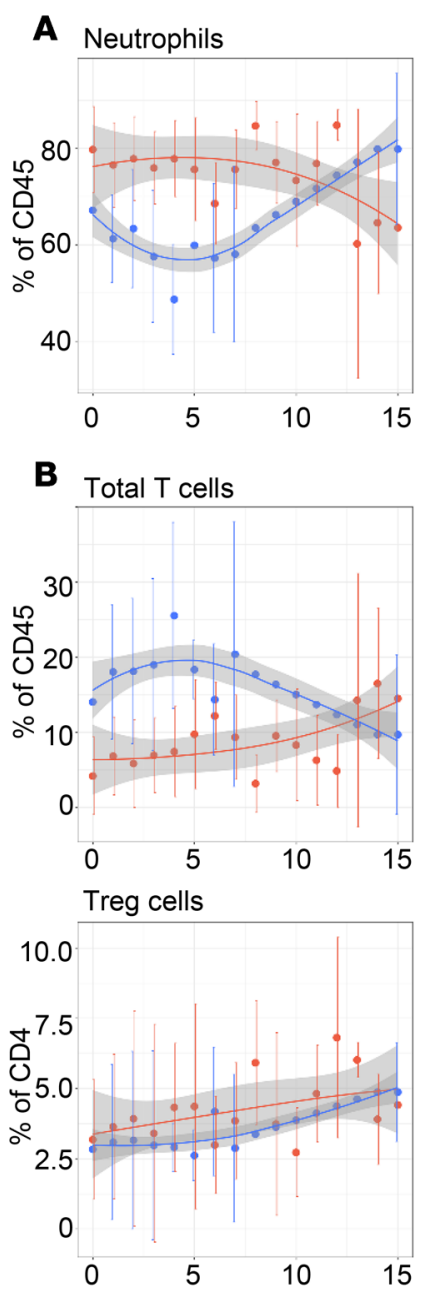
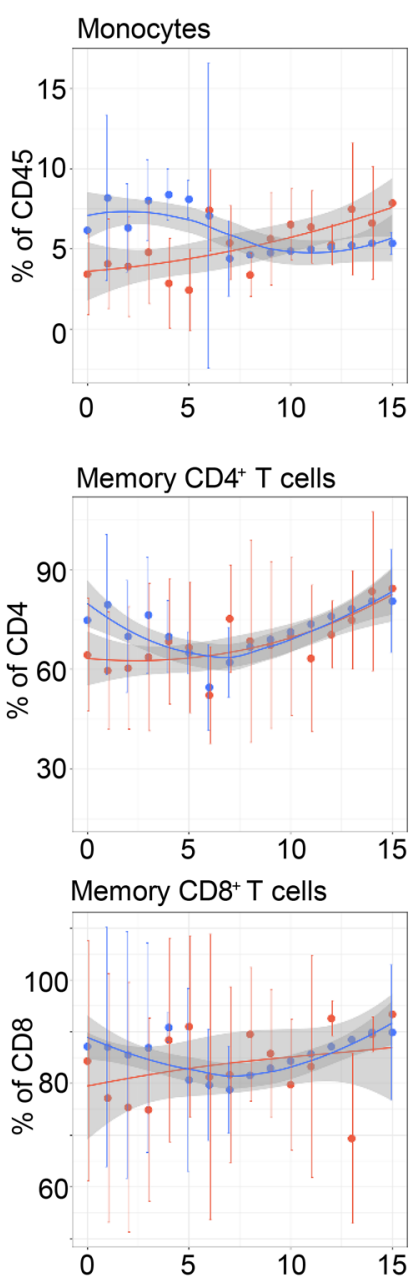
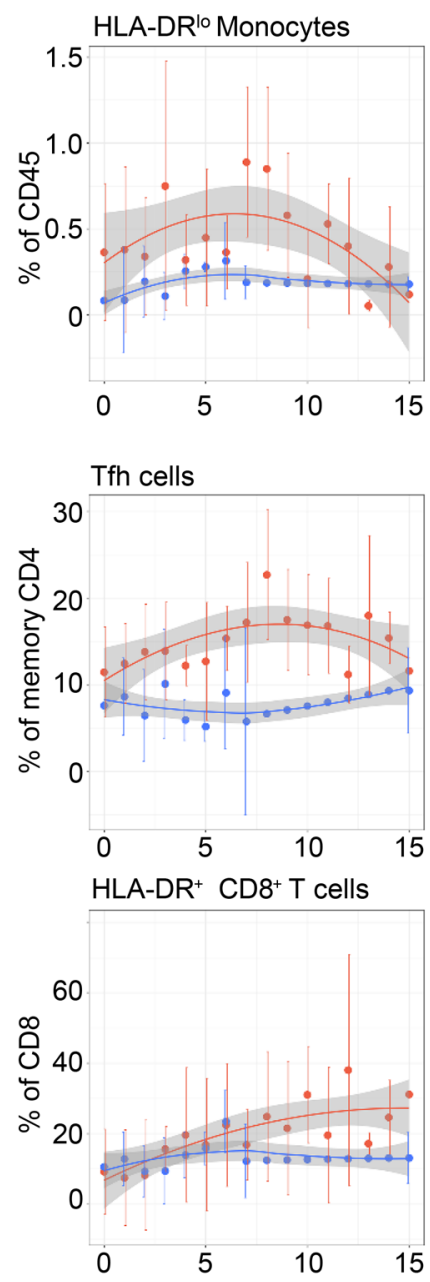
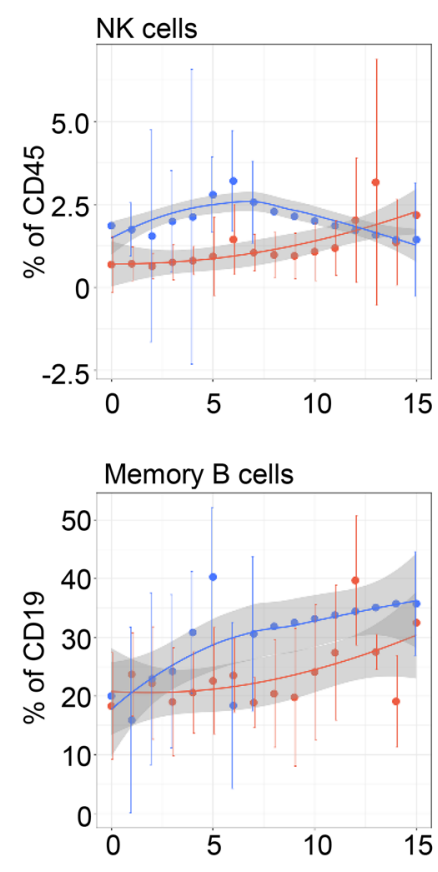

Moderate COVID-19

Severe COVID-19

Figure 8. Immune profiles of moderate and severe patients diverge over time, reflecting different disease trajectories. Longitudinal plots of gated populations for (A) innate and (B) adaptive cell types. Days (relative) from first hospitalization are shown. Loess trajectory smoothing was performed on the median values (colored disks) for each group at each time point. Vertical bars indicate \pm 1 SD around the median at each time point. Plot points without vertical error bars are from single data points, or interpolated values used for smoothing.

higher diabetes prevalence than the US or Washington rates (28); the highest prevalence in Washington state is among 65-74 year olds at $21.5 \%$, which is more than doubled in the cohort with severe disease described here. Diabetes and obesity have consistently been identified as risk factors for COVID-19 severity (4-9); reduced $\mathrm{T}$ cell function and chronic inflammation have been postulated as potential mechanisms driving this increased risk (29). In addition, some glucose-lowering agents used in diabetes are known to impact the immune system (reviewed in ref. 30). Full analysis of the differential impact of diabetes and its treatment on our immune signatures is beyond the scope of this work but merits further analysis.

There are limitations to this study. Due to the urgency of the pandemic, we chose to use recovered clinical samples for our study and thus the collection schedule and sample availability was dictated by the treatment needs of the patient. This meant that we did not have the same time points for every patient, and that we could not match between groups the medications that individuals were already taking due to preexisting comorbidities, some of which may impact the immune responses seen here. In addition, the differences between the mild, moderate, and severe COVID-19 groups may reflect the time from disease onset, which significantly varied between these groups, and/or differences in viral burden, which we could not assess. The subjects in our hospitalized control group were not matched to the SARS-CoV-2-positive groups by race, although they are well matched by age.

In summary, we have identified unique features of the immune landscape in moderate versus severe COVID-19 along with features that are common to moderate and severe non-COVID-19 illness. Importantly, our findings indicate that selection of immune interventions should be based in part on disease presentation and early disease trajectory due to the profound differences in the immune response in those with mild to moderate disease and those with the most severe disease. Finally, our characterization of the variety of immune signatures in COVID-19 provides insight into the types of immune interventions that may be beneficial in the treatment of severe disease. 
A

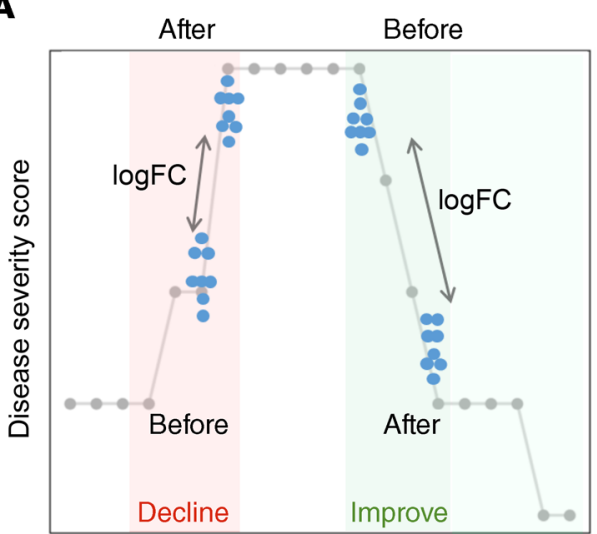

Days from admission
B

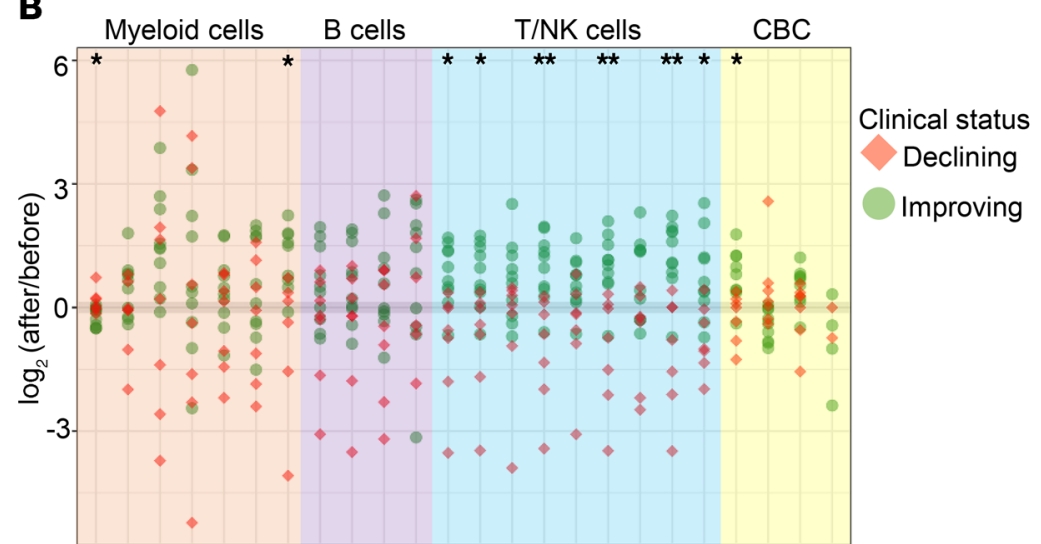

C
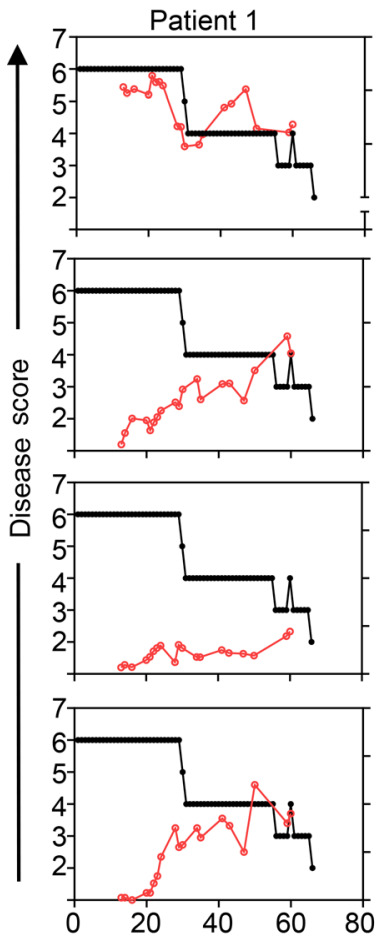
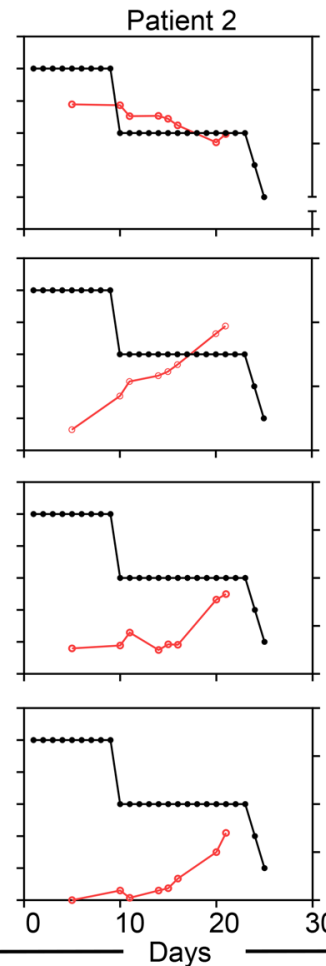

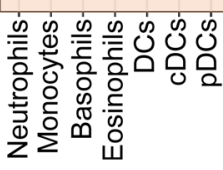

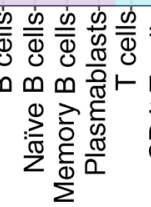

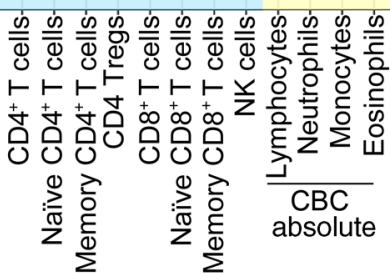

Patient 3

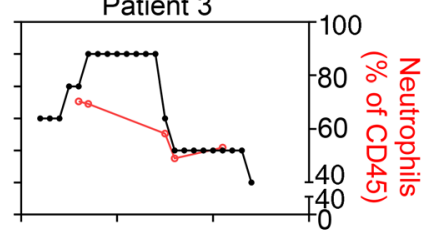

30

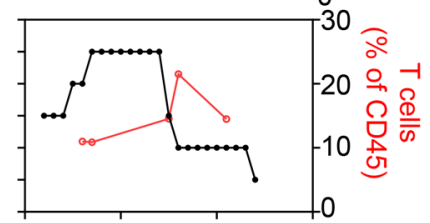

(a) 을 吕

๙०

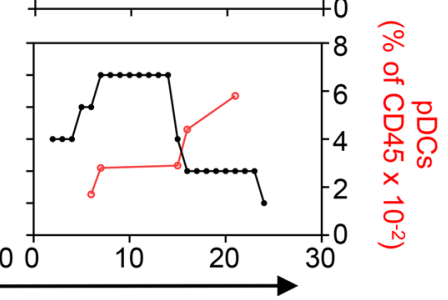

Figure 9. Immune signatures of clinical decline and improvement. (A) Schematic outlining approach for identifying immune signatures of clinical decline and improvement focusing on changes in monitored parameters in longitudinal samples taken before and after changes in clinical score. (B) $\log _{2}$-fold change in the indicated cell populations as measured by CyTOF or CBC analysis in longitudinal samples taken before and after improving (green) or declining (red) clinical scores. Asterisks indicate a significant difference in the fold changes (2-tailed, unpaired Wilcoxon rank sum FDR-adjusted $P<0.05)$ between improving $(n=7)$ and declining $(n=10)$ patient groups for the indicated cell populations. (c) Longitudinal analyses of the frequency of neutrophils, $T$ cells, NK cells, and pDCs vs. clinical score in 3 individual patients. Black line shows disease score (left $y$ axis) and red line shows immune cell frequency (right $y$ axis).

\section{Methods}

Study design. Using our newly developed 33-parameter CyTOF panel, we characterized the immune response longitudinally in 59 adults with acute COVID-19, including 24 hospitalized patients with severe disease, 28 hospitalized patients with moderate disease, and 7 ambulatory patients with mild disease not requiring hospitalization. All COVID-19 subjects were positive for SARS-CoV-2 and our control cohort of 17 hospitalized patients tested negative for SARS-CoV-2. Healthy control subjects were age- and sex-matched to the hospitalized COVID-19 subjects. Importantly, the samples used were collected prior to the start of the COVID-19 pandemic in December 2019. For the hospitalized COVID-19 cohort, longitudinal samples were collected starting as soon as possible after hospital admission, then if feasible, daily for the first week, and then at 3-4 day intervals (Figure 1). A single sample was obtained at time of first outpatient visit for the ambulatory COVID-19 subjects. A maximum of 2 samples was obtained from the hospitalized COVID-19-negative control subjects. All assays were run and analyzed in a blinded manner.

CyTOF staining, acquisition, and subset identification. Peripheral blood was collected from each donor into sterile vacutainer tubes containing the anticoagulant EDTA. Blood cells were washed twice with PBS and stained for viability exclusion with a $100 \mu \mathrm{M}$ cisplatin solution (Enzo Life Sciences) for 1 minute at room temperature. Cisplatin was quenched with 5 volumes MaxPar Cell Staining Buffer (CSB; Fluidigm), 
A
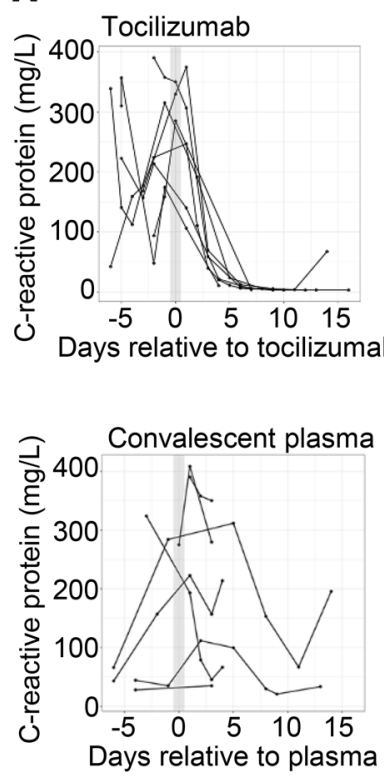

D
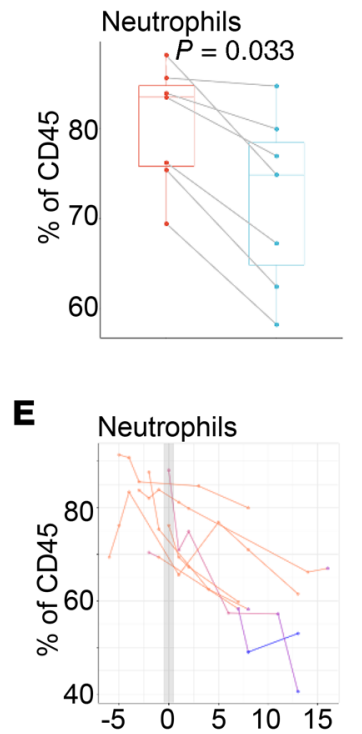

B

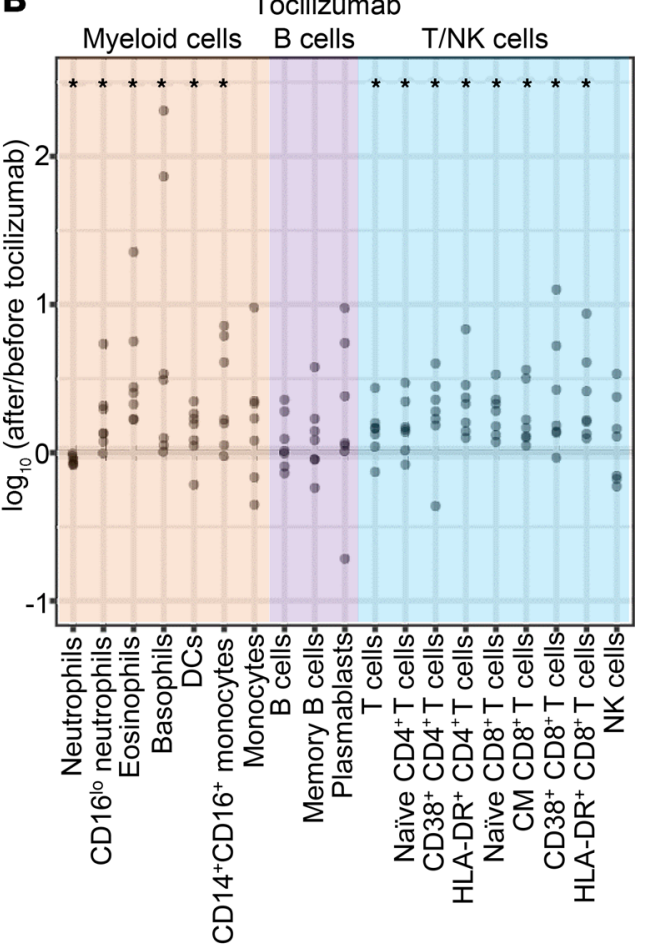

C Convalescent plasma

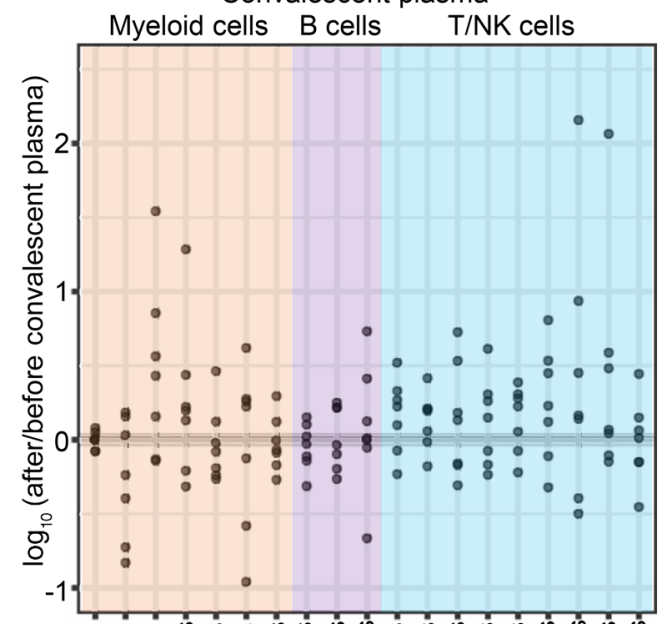

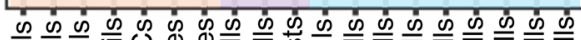

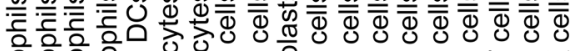

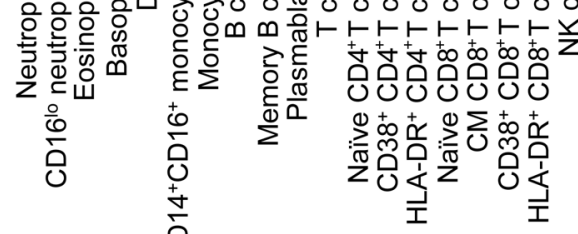
HLA-DR+ CD8 $8^{+}$cells 50 $P=0.033$
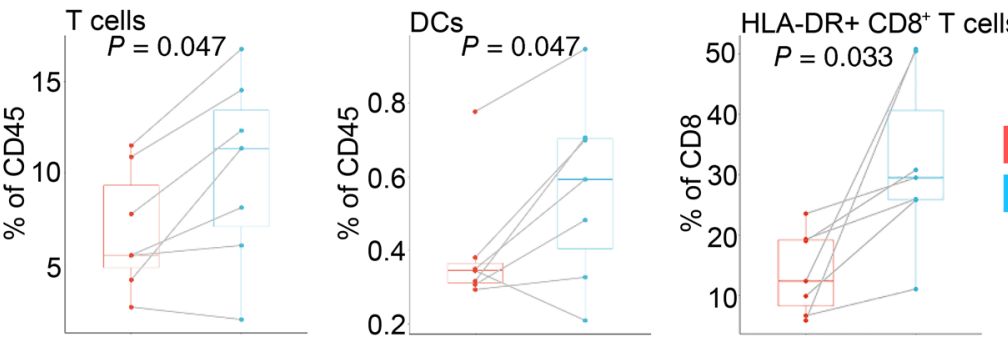

Before tocilizumab

After tocilizumab
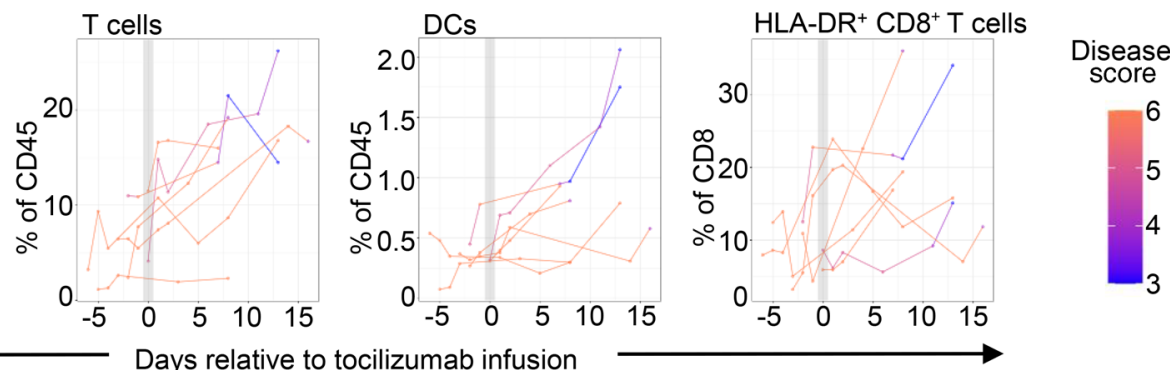

Figure 10. Early immune responses to tocilizumab but not convalescent plasma in severe COVID-19. (A) Serum CRP in patients receiving tocilizumab (top; $n=7$ ) or convalescent plasma (bottom; $n=7$ ) measured in clinical labs relative to day of treatment. Each line represents an individual patient. (B and C) Change in blood immune populations measured by CyTOF after treatment with tocilizumab (B) or convalescent plasma (C). The fold change in each population for each subject was determined by dividing the percentage of each population in the first posttreatment sample at day +2 or more after treatment with the closest pretreatment sample available as detailed in Supplemental Table 3. All are shown as percentage of CD45 cells unless otherwise indicated. (D) Plots showing the percent of the indicated populations in tocilizumab-treated patients before and after treatment, using the time points used for analysis in B and Supplemental Table 3. (E) Plots showing all the data points available for tocilizumab-treated patients for the indicated populations shown in $\mathbf{D}$ and Supplemental Table 3. Each line represents an individual patient and the color of the line reflects the clinical ordinal score at the time of sampling. ${ }^{*} P<0.05$ Wilcoxon matched pairs test, adjusted for multiple comparisons.

and the cells then stained with a titered, aliquoted, and frozen cocktail of monoclonal antibodies conjugated to metal isotopes for 20 minutes at $4^{\circ} \mathrm{C}$. Red blood cell lysis was performed using RBC Lysis/Fixation solution (BioLegend) for 5 minutes at room temperature followed by a wash with CSB. The resulting leukocytes were fixed overnight at $4^{\circ} \mathrm{C}$ with MaxPar Fix and Perm Solution (Fluidigm) containing 125 nM Cell-ID Intercalator-Ir (Fluidigm). Following fixation, cells were washed with CSB, resuspended in milli-Qwater, and stored at $4^{\circ} \mathrm{C}$ until 
acquisition. All antibodies from BioLegend and BD Biosciences were conjugated to their respective metal isotopes using the MaxPar X8 Multimetal Labeling Kit (Fluidigm). Samples were stained within 48 hours of blood draw. Sample stability with the CyTOF assay was established with 3 COVID-19 samples assayed on the day of collection (baseline, day 0), one day after collection (day 1) or 2 days after collection (day 2). Populations and markers on populations were standard and based on Staser et al. (31). All populations and markers with a frequency greater than $5 \%$ of live CD $45^{+}$cells had a CV less than $40 \%$ between baseline and each time point. This variation was less than that of biological comparisons. MaxPar Four Element Calibration Beads (Fluidigm) were added to each sample immediately before acquisition. All samples were acquired on a Helios CyTOF mass cytometer (Fluidigm) with a target cell acquisition of 100,000 live events at a rate of 500 events/second to capture greater than 50 cells per gated population or marker. The CyTOF panel is shown in Supplemental Table 1 and gating strategies are shown in Supplemental Figures 2-4. To determine gates for activation markers such as CD25, CD69, and CD38 on T cells and PD-L1 on myeloid cells, we first analyzed 12 samples from 6 subjects with moderate COVID-19 and 6 subjects with severe COVID-19. Gates were set based on a comparison between samples that were clearly highly activated and those that were clearly nonactivated. These gates were then applied to all samples in the study and used consistently for all populations analyzed. Specifically, gates for CD25, CD38, CD69, HLA-DR, PD-1, and PD-L1 were the same for all cell types where they were applied. For example, the CD38 gate was the same for $\mathrm{CD} 4^{+} \mathrm{T}$ cells, CD8 ${ }^{+} \mathrm{T}$ cells and Tfh cells, the $\mathrm{CD} 25$ gate was the same for $\mathrm{CD} 4^{+}$ and $\mathrm{CD}^{+} \mathrm{T}$ cells, and the CD69 gate was the same for CD4 ${ }^{+} \mathrm{T}$ cells, $\mathrm{CD}^{+} \mathrm{T}$ cells, NK cells, eosinophils, neutrophils, etc. Data were analyzed using a FlowJo software versions 10.6.0 and 10.6.1 (FlowJo).

Statistics. Apart from the paired-sample tests in Figure 10, all $P$ values were calculated using unpaired, 2-tailed Wilcoxon rank sum tests. In all cases, corrections for multiple testing were performed using the FDR method. For between group comparisons of the clinical data, $P$ values were calculated using the Kruskal-Wallis 1-way analysis of variance test. The correlation graph in Figure 2A was built from the matrix of Pearson correlations in Figure 2B using the RiGraph package (32). The heatmap in Figure 6 was generated using Euclidean distance and the clustering method Ward.D2.

Time-series data from each patient were organized in terms of the relative number of days from the date of the first sample (hereon denoted pseudo-time), and then aligned by first sample. To reduce the potential effects of outlier samples, median values were calculated for each severity category and each day for the samples available. If no samples were available at a given pseudo-time day, we inferred a value using linear interpolation between the before and after pseudo-time points. The vertical bars at each pseudo-time point are equal to 1 standard deviation from the indicated median value. Plot points with no error bars are those with only 1 sample or represent an inferred value. Loess smoothing was performed on the median values for each disease severity class using the geom smooth function in the R ggplot library (33).

The UMAP plots in Figure 7 were generated directly from the CyTOF signal intensities following archsinh transformation with a cofactor value of 5 . To ensure against batch and other potential confounding effects, we specifically selected samples collected and stained in a highly uniform fashion from a single donor and $z$ score normalized probe intensities for each sample prior to UMAP projection to $2 \mathrm{D}$.

Study approval. Samples from COVID-19 subjects and from hospitalized COVID-19-negative control subjects were recovered from the Virginia Mason Medical Center Central Processing Lab after all tests required for clinical care were complete, under approval by the Benaroya Research Institute (BRI) protocol IRB20-036. All healthy control samples were from healthy subjects in the BRI Immune-Mediated Disease Registry and Repository who had given written informed consent in accordance with the Declaration of Helsinki and according to the BRI IRB-approved protocol IRB07109.

\section{Author contributions}

SAL, CS, UM, JAH, DJC, and JHB conceptualized and designed the study. UM and JHB were responsible for subject selection and clinical interpretation. The BRI COVID-19 Research Team obtained IRB approval, collected samples and clinical data, conducted the mass cytometry, and integrated the clinical data with the CyTOF data. SAL, DS, and JAH performed the gating for the immune cell populations measured by CyTOF. HB, SAL, DS, and JAH performed the computational and statistical analysis. JAH, AMH, and JHB wrote the manuscript with assistance from all coauthors. JHB obtained funding and supervised the study.

\section{Acknowledgments}

We would like to acknowledge the Benaroya Family Foundation, the Leonard and Norma Klorfine Foundation, and Glenn and Mary Lynn Mounger for their funding of this project. This work was supported by NIH grants R01AI150178-01S1 (to JAH) and U19AI125378-05S1 (to DJC and JAH). We acknowledge the Allen Institute for Immunology for their funding support for the development of the CyTOF panel used in this study. We also would like to thank Carmen Mikacenic for help with obtaining IRB approval, and Henry T. Bahnson for help with statistical analysis. See Supplemental Acknowledgments for the BRI COVID-19 Research Team details. The graphical abstract was created with BioRender.com.

Address correspondence to: Jane H. Buckner, Benaroya Research Institute at Virginia Mason, 1201 Ninth Avenue, Seattle, WA 98101. Phone: 206.287.1033; Email: jbuckner@benaroyaresearch.org.
1. Williamson EJ, et al. Factors associated with COVID-19-related death using OpenSAFELY. Nature. 2020;584(7821):430-436.

2. Barron $\mathrm{E}$, et al. Associations of type 1 and type 2 diabetes with COVID-19-related mortality in England: a whole-population study. Lancet Diabetes Endocrinol. 2020;8(10):813-822.

3. Holman N, et al. Risk factors for COVID-19-related mortality in people with type 1 and type
2 diabetes in England: a population-based cohort study. Lancet Diabetes Endocrinol. 2020;8(10):823-833.

4. Shi $\mathrm{Q}$, et al. Clinical characteristics and risk factors for mortality of COVID-19 patients with diabetes in Wuhan, China: a two-center, retrospective study. Diabetes Care. 2020;43(7):1382-1391.

5. Cai Q, et al. Obesity and COVID-19 severity in a designated hospital in Shenzhen, China. Diabetes
Care. 2020;43(7):1392-1398.

6. Chen Y, et al. Clinical characteristics and outcomes of patients with diabetes and COVID-19 in association with glucose-lowering medication. Diabetes Care. 2020;43(7):1399-1407.

7. Singh AK, et al. Diabetes in COVID-19: Prevalence, pathophysiology, prognosis and practical considerations. Diabetes Metab Syndr. 2020;14(4):303-310. 
8. Kim L, et al. Risk factors for intensive care unit admission and in-hospital mortality among hospitalized adults identified through the U.S. coronavirus disease 2019 (COVID-19)-associated hospitalization surveillance network (COVIDNET). Clin Infect Dis. 2020; ciaa1012.

9. Gao F, et al. Obesity is a risk factor for greater COVID-19 severity. Diabetes Care. 2020;43(7):e72-e74.

10. Price-Haywood EG, et al. Hospitalization and mortality among black patients and white patients with Covid-19. N Engl JMed. 2020;382(26):2534-2543.

11. Karaca-Mandic P, et al. Assessment of COVID-19 hospitalizations by race/ethnicity in 12 states. JAMA Intern Med. 2020; 203857.

12. Cao B, et al. A trial of lopinavir-ritonavir in adults hospitalized with severe covid-19. N Engl J Med. 2020;382(19):1787-1799.

13. United States Census Bureau. QuickFacts Washington. https://www.census.gov/ quickfacts/WA. Updated December 7, 2020. Accessed September 16, 2020.

14. Chen $\mathrm{G}$, et al. Clinical and immunologic features in severe and moderate coronavirus disease 2019. J Clin Invest. 2020;130(5):2620-2629.

15. Qin C, et al. Dysregulation of immune response in patients with coronavirus 2019 (COVID-19) in Wuhan, China. Clin Infect Dis. 2020;71(15):762-768. 16. Liu J, et al. Neutrophil-to-lymphocyte ratio predicts critical illness patients with 2019 coronavirus disease in the early stage. J Transl Med.2020;18(1):206.

17. Zhang B, et al. Immune phenotyping based on the neutrophil-to-lymphocyte ratio and IgG level predicts disease severity and outcome for patients with COVID-19. Front Mol Biosci. 2020;7:157.

18. Kuri-Cervantes L, et al. Comprehensive mapping of immune perturbations associated with severe COVID-19. Sci Immunol. 2020;5(49):eabd7114.

19. Laing AG, et al. A dynamic COVID-19 immune signature includes associations with poor prognosis. Nat Med. 2020;26(10):1623-1635.

20. Rodriguez L, et al. Systems-level immunomonitoring from acute to recovery phase of severe COVID-19. Cell Rep Med.2020;1(5):100078.

21. Giamarellos-Bourboulis EJ, et al. Complex immune dysregulation in COVID-19 patients with severe respiratory failure. Cell Host Microbe. 2020;27(6):992-1000.e3.

22. Lukaszewicz AC, et al. Monocytic HLA-DR expression in intensive care patients: interest for prognosis and secondary infection prediction. Crit Care Med. 2009;37(10):2746-2752.

23. Davenport EE, et al. Genomic landscape of the individual host response and outcomes in sepsis: a prospective cohort study. Lancet Respir Med. 2016;4(4):259-271.

24. Faivre V, et al. Downregulation of blood monocyte HLA-DR in ICU patients is also present in bone marrow cells. PLoS One. 2016;11(11):e0164489.
25. Lucas C, et al. Longitudinal analyses reveal immunological misfiring in severe COVID-19. Nature. 2020;584(7821):463-469.

26. Mathew D, et al. Deep immune profiling of COVID-19 patients reveals distinct immunotypes with therapeutic implications. Science. 2020;369(6508):eabc8511.

27. Rosas I, et al. Tocilizumab in hospitalized patients with COVID-19 pneumonia [preprint]. https://doi.org/10.1101/2020.08.27.20183442. Posted on medRxiv September 12, 2020.

28. Centers for Disease Control and Prevention. CDC COVID Data Tracker. 2020. https://covid. cdc.gov/covid-data-tracker/.

29. Muniyappa R, Gubbi S. COVID-19 pandemic, coronaviruses, and diabetes mellitus. Am JPhysiol Endocrinol Metab. 2020;318(5):E736-E741.

30. Drucker DJ. Coronavirus infections and type 2 diabetes-shared pathways with therapeutic implications. Endocr Rev. 2020;41(3):bnaa011.

31. Staser KW, et al. OMIP-042: 21-color flow cytometry to comprehensively immunophenotype major lymphocyte and myeloid subsets in human peripheral blood. Cytometry A. 2018;93(2):186-189.

32. Csardi G, Nepusz T. The igraph software package for complex network research. InterJournal. Complex Systems, 1695. 2006. https://igraph. org. Accessed December 8, 2020.

33. Wickham H. ggplot2: Elegant Graphics for Data Analysis. Springer-Verlag; 2016. 